\title{
MUMPS OUTBREAK ONGOING SINCE OCTOBER 2007 IN the RePublic of Moldova
}

\author{
H Bernard (bernardh@rki.de)1,2, N G Schwarz ${ }^{3,4}$, A Melnic 5 , V Bucov5 ${ }^{5}$ N Caterinciuc ${ }^{5}$, R G Pebody $^{6,4}$, M Mulders ${ }^{7}$, C Aidyralieva7, \\ S Hahné $\mathbf{p}^{7,8}$ \\ 1. Robert Koch Institut (RKI), Berlin, delegated to Bavarian Health and Food Safety Authority (LGL), Oberschleissheim, Germany \\ 2. Postgraduate Training for Applied Epidemiology (PAE, German Field Epidemiology Training Program, FETP), Germany \\ 3. Institut de Veille Sanitaire (French National Institute of Health, InVS), Paris, France \\ 4. European Training Programme for Intervention Epidemiology (EPIET), European Centre for Disease Prevention and Control (ECDC), \\ Stockholm, Sweden \\ 5. National Scientific and Practical Centre of Preventive Medicine (NSPCPM), Chisinau, Republic of Moldova \\ 6. Health Protection Agency (HPA), London, United Kingdom \\ 7. World Health Organization (WHO) Regional Office for Europe, Copenhagen, Denmark \\ 8. Rijksinstituut voor Volksgezondheid en Milieu (National Institute for Public Health and the Environment, RIVM), Bilthoven, The \\ Netherlands
}

The Republic of Moldova is experiencing a nationwide mumps outbreak, with a total of 19,550 notified cases as of 23 March 2008.

The outbreak started in October 2007, with 105 cases notified in that month compared to an average number of 24 cases per month notified between January and September 2007. Between 1 October and 31 December 2007, 1,524 cases were notified.

In the Republic of Moldova, mumps monovalent vaccination was introduced in 1983 as a single-dose schedule targeting children aged 15-18 months-old. Since 2002, a second dose has been administered to six- to seven-year-olds (birth cohorts from 1995 onwards), using a combined vaccine for measles, mumps, and rubella (MMR). Reported mumps vaccination coverage has been high since its introduction, except for a period in the early 1990s when a vaccine shortage occurred due to political changes. Successful catch-up campaigns subsequently improved vaccination coverage in those birth cohorts affected by the vaccine shortage. According to routine national surveillance data, coverage with one dose of mumps vaccine is $\geq 94 \%$ in individuals born in 1989-1993. Coverage with two doses is $\geq 96 \%$ in individuals born in 1995-2000. Coverage in the 1994 birth cohort is $99 \%$ and $21 \%$ with one and two doses, respectively. The most recent large mumps outbreak in the Republic of Moldova occurred in 1996-1998, with 28,845 cases reported, predominantly from birth cohorts 1983-1990 (60\%).

In February 2008, the country's Ministry of Health invited an international outbreak investigation team coordinated by the World Health Organization Regional Office for Europe, to identify the extent of the mumps outbreak and assess cases and their possible risk factors for acquiring mumps, and to provide recommendations for the current outbreak.

The following is a preliminary description of the extent and characteristics of the outbreak until 2 March.

\section{Methods}

In the Republic of Moldova, mumps cases are notified by family doctors, hospitals and health centres to 44 Regional Centres for Preventive Medicine (RCPMs), with date of onset, initial diagnosis, hospitalisation, and vaccination history where available. The RCPMs aggregate and transmit the data to the National Scientific and Practical Centre for Preventive Medicine (NSPCPM) on a monthly and annual basis. In addition to the routine communicable diseases reporting, a weekly transmission of mumps data to the NSPCPM was introduced on 17 December 2007 to monitor the outbreak. These reports are based on the emergency notifications of suspected cases and contain information on age, vaccination status, hospitalisation, and number of cases in educational institutions. Here we describe the cases reported weekly between 17 December 2007 and 2 March $2008(n=13,853)$.

Mumps is notified when suspected on clinical grounds by a physician. For a minority of cases $(n=388,3 \%)$, laboratory testing was carried out. Mumps IgM antibody testing in patients' sera ( $n=367$ ) was performed at the NSPCPM using a mumps IgM ELISA kit (Novalisa, Novatec Immundiagnostica GmbH, Germany). Polymerase chain reaction (PCR) of clinical specimens $(n=21)$ including throat swabs, oral fluid, and urine, was performed at the National Institute for Public Health and the Environment (RIVM) in the Netherlands, and at the Health Protection Agency (HPA), Centre for Infections, in the United Kingdom (UK).

\section{Outbreak description}

Between 1 October 2007 and 2 March 2008, a total of 14,729 mumps cases were notified in the Republic of Moldova. Case notifications increased rapidly in the weeks following the holidays around New Year's Day. The outbreak is ongoing at the time of writing this report, with a peak of 2,096 cases in week 9 of 2008 (Figure 1). The monthly incidence increased from below one case per 100,000 population in the months preceding the outbreak to 25 cases per 100,000 in December 2007 and over 170 cases per 100,000 in February 2008. 


\section{Case description}

The majority of cases ( $n=11,128 ; 80 \%)$ were 15 to 24 years old (birth cohorts 1983-1992), and 60\% $(n=8,298)$ were male (Figure 2). By the end of February, all regions of the Republic of Moldova were affected by the outbreak.

Vaccination data were available for $67 \%(9,223$ of 13,853 cases) and $96 \%$ of them had been vaccinated. Of these, $96 \%$ had received one dose of mumps vaccine and $4 \%$ had received two doses.

In total, 5,649 cases (41\%) were hospitalised for epidemiological (isolation) and clinical indications, and where care was not available (e.g. students living in dormitories). Information about complications was not available at the national level. No deaths have been reported.

Of 367 serum samples taken from clinical cases (one to 28 days after onset of parotid swelling), 234 (64\%) were positive for antimumps IgM at the NSPCPM. 313 of the 367 sera were collected eight to 28 days after disease onset, $68 \%$ of which were positive. The highest proportion of positive results was found in the sera collected between 11 and 20 days after disease onset (72\%). Of 21 case sera tested with PCR at RIVM (three cases) and HPA (18 cases), 20 were positive for mumps virus. The identified genotype G5 has recently been found in several other countries including Croatia, Denmark, Germany, the UK, Canada, and the United States (US) (personal communication with Dr K. Brown/Dr L. Jin, HPA).

Public health measures

For this outbreak, the NSPCPM recommended hospitalisation for epidemiologic indications of those cases for whom isolation was not guaranteed at home, particularly for cases living in dormitories and boarding schools. Recommended isolation was 10 days from onset of parotid swelling.

In January 2008, the Ministry of Health and the NSPCPM initiated the procurement of 600,000 doses of MMR vaccine for a Supplemental Immunisation Activity (SIA). As of 6 March 2008, a total of 42,500 doses had arrived in the Republic of Moldova and were distributed to health facilities. The SIA began in week 9 of 2008 targeting individuals in all settings born between 1989 and 1994 (including pupils, students and postgraduate students in all educational institutions; teaching staff born between 1984 and 1988; army, police, and border troops). Information on the total number of MMR vaccine doses administered to date during the SIA is not available.

\section{Discussion}

The Republic of Moldova is facing a large ongoing mumps outbreak in teenagers and young adults, the majority of whom have previously been vaccinated with one dose of monovalent mumps vaccine. Mumps outbreaks comparable to this one with respect to size and affected age group have occurred recently in several countries, including the UK, the US, and Canada. In the outbreak in the UK $[1,2,3], 79 \%$ of cases in 2004 occurred in individuals born between 1980-1989. Almost two thirds of the cases were unvaccinated, however, $30 \%$ had received a single dose of MMR. In the outbreaks in the US and Canada [4], 49\% of the cases had been vaccinated twice. Mumps vaccine failure has often been attributed to primary vaccine failure, i.e. an insufficient immune response after vaccination [5]. More recent studies have suggested that secondary vaccine failure, i.e. decreasing antibody titres over
F I G U R E 1

Mumps cases

A) by week (17 December 2007 to 2 March 2008, n=13,853) and B) by month (July 2007 to February 2008, $n=14,438$ ) of notification, Republic of Moldova*
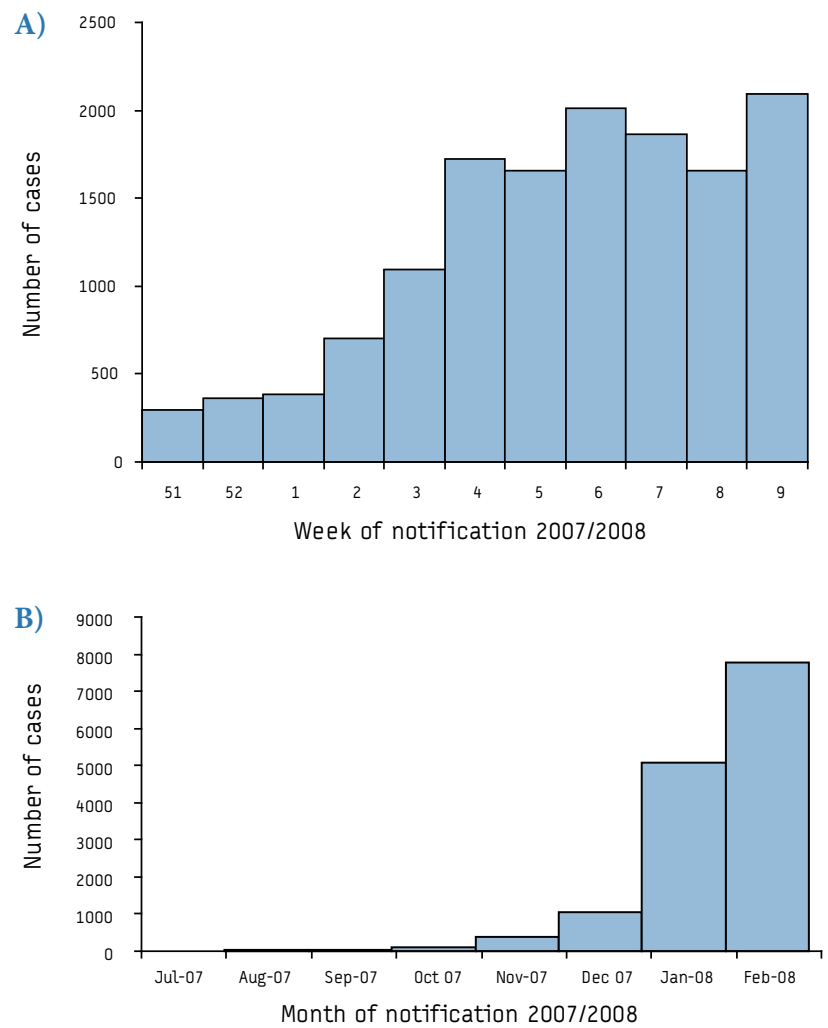

* Data source: NSPCPM, Republic of Moldova

F I G U R E 2

Mumps cases with known vaccination status by aggregated birth cohorts, vaccination status, and vaccination schedules corresponding to birth cohorts*, Republic of Moldova, 17 December 2007 to 2 March $2008^{* \star}(n=9,223)$

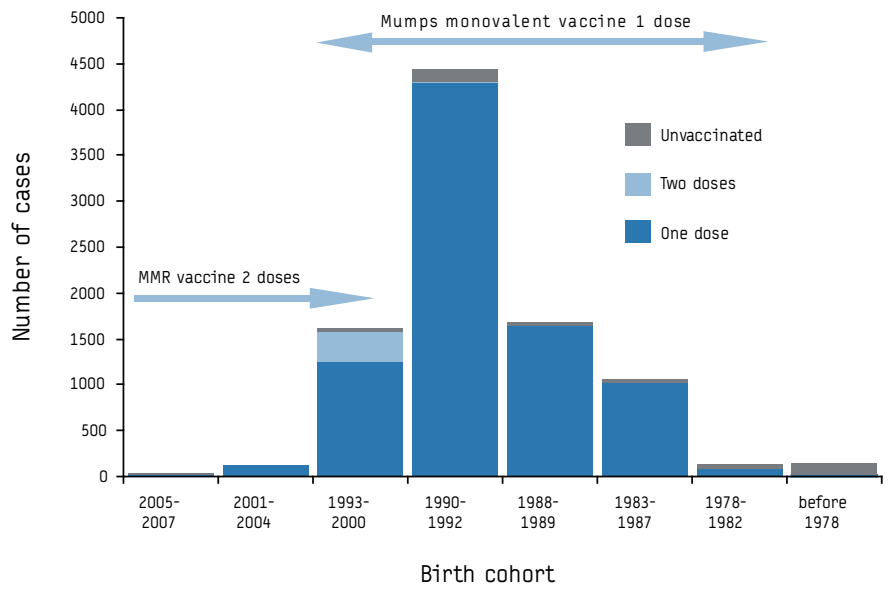

* Subdivided data on vaccination schedule for cases in birth cohorts 1993-2000 and 1978-1982 not available at the national level ** Data source: NSPCPM, Republic of Moldova 
time (waning immunity), contributes to the recurrence of mumps outbreaks $[6,7]$. In the current outbreak, the high reported vaccine coverage with a single dose of mumps vaccine among the cases suggests that "failure to vaccinate" did not play a major role as the cause of the outbreak. Primary and secondary vaccine failure $[5,8]$ for one dose of mumps vaccine are currently being investigated in a further epidemiological study.

A high proportion of cases in this outbreak were hospitalised, mainly to ensure isolation but also for clinical and social indications. However, as mumps is most infectious from two days before to four days after onset of illness, and inapparent infections can be communicable, hospital admission is not expected to prevent the majority of transmission in the population [9].

Outbreak control is ongoing with vaccination of high risk groups with MMR vaccine, consistent with a recent WHO position paper on mumps [10]. To address potential concerns of adverse events following immunisation (AEFI), AEFI surveillance including laboratory investigation of suspected meningitis cases and training of primary health care staff and epidemiologists will accompany this immunisation campaign.

\section{Acknowledgements}

The authors would like to thank the staff of the RCPMs for providing the data, Dr R van Binnendijk (RIVM), Dr K Brown (HPA) and Dr L Jin for providing laboratory data, Dr $\vee$ Bremer (ECDC/EPIET) for helpful comments on the manuscript, T Gavrilenco for translating, the Ministry of Health of the Republic of Moldova, Dr I Bahnarel (NSPCPM), and Dr P Ursu (WHO Country Office, Republic of Moldova) for their support during the mission.

Note: H Bernard and NG Schwarz contributed equally to this article.

\section{References}

1. Cohen C, White JM, Savage EJ, Glynn JR, Choi Y, Andrews N, et al. Vaccine effectiveness estimates, 2004-2005 mumps outbreak, England. Emerg Infect Dis. 2007;13(1):12-7.

2. Savage E, Ramsay M, White J, Beard S, Lawson H, Hunjan R, et al. Mumps outbreaks across England and Wales in 2004: observational study. BMJ. 2005;330(7500):1119-20.

3. Peltola H, Kulkarni PS, Kapre SV, Paunio M, Jadhav SS, Dhere RM. Mumps outbreaks in Canada and the United States: time for new thinking on mumps vaccines. Clin Infect Dis. 2007;45(4):459-66.

4. Centers for Disease Control and Prevention. Mumps epidemic - United Kingdom 2004-2005. MMWR Morb Mortal Wkly Rep. 2006; 55(7):173-5. Available from: http://www.cdc.gov/mmwr/preview/mmwrhtml/mm5507a1.htm

5. Briss PA, Fehrs LJ, Parker RA, Wright PF, Sannella EC, Hutcheson RH, et al. Sustained transmission of mumps in a highly vaccinated population: assessment of primary vaccine failure and waning vaccine-induced immunity. J Infect Dis. 1994;169(1):77-82.

6. Park DW, Nam MH, Kim JY, Kim HJ, Sohn JW, Cho Y, et al. Mumps outbreak in a highly vaccinated school population: assessment of secondary vaccine failure using IgG avidity measurements. Vaccine. 2007;25(24):4665-70.

7. Vandermeulen C, Roelants M, Vermoere M, Roseeuw K, Goubau P, Hoppenbrouwers $K$. Outbreak of mumps in a vaccinated child population: a question of vaccine failure? Vaccine 2004;22(21-22):2713-6.

8. Hersh BS, Fine PE, Kent WK, Cochi SL, Kahn LH, Zell ER, et al. Mumps outbreak in a highly vaccinated population. J Pediatr. 1991;119(2):187-93.

9. Robertson S. Mumps. In: Heymann DL, editor. Control of Communicable Diseases Manual. 18th ed. The American Public Health Association; 2004. p. 409-13.

10. WHO. Mumps virus and vaccines. Weekly epidemiological record 2007;82(7):51-60. Available from: http://www.who.int/entity/wer/2007/wer8207.pdf
This article was published on 27 March 2008.

Citation style for this article: Bernard H, Schwarz NG, Melnic A, Bucov V, Caterinciuc N, Pebody RG, Mulders M, Aidyralieva C, Hahné S. Mumps outbreak ongoing since October 2007 in the Republic of Moldova. Euro Surveill. 2008;13(13):pii=8079. Available online: http://www.eurosurveillance.org/ViewArticle.aspx?ArticleId=8079 